\title{
PATH DEPENDENCE (TRAYECTORIAS DEPENDIENTES) EN LA MATRIZ ELÉCTRICA DE COSTA RICA
}

PATH DEPENDENCE IN COSTA RICA'S ELECTRIC MATRIX

\author{
Daniel Francisco Avendaño Leadem¹, Daniela García Sánchez \\ ${ }^{1}$ Universidad Nacional de Costa Rica (UNA), Heredia, Costa Rica
}

Correspondência para: Daniel Francisco Avendaño Leadem (davenda@una.cr)

doi: 10.12957/geouerj.2019.44815

Recebido em: 26 ago. 2019 | Aceito em: 17 set. 2019

\section{RESUMEN}

Los intereses de investigación comunes entre estudios en geografía y energía representan una oportunidad para llevar a cabo un análisis profundo que permita comprender la relación que existe entre las diferentes trayectorias de transición de energía y las condiciones geográficas de las cuales emergen. Considerando a Costa Rica como un caso de estudio empírico y en aplicación de teorías compartidas entre los campos de geografía económica y teorías neo-institucionales, esta investigación analiza el desarrollo de la matriz energética costarricense, mediante la identificación de tres formas de mecanismos de path dependence (trayectorias dependientes): lock-in tecnológico externalidades positivas e histéresis institucionales. Considerando un sector eléctrico que es casi $100 \%$ renovable y con el compromiso nacional de alcanzar la carbono neutralidad, este artículo provee claridad hacia el entendimiento de un camino que es difícil de revertir a pesar de los problemas que rodean la explotación de los recursos convencionales. Ofrece un ejemplo práctico de aplicación de una teoría económica y geográfica desde una perspectiva evolutiva con miras a lograr una interpretación de la progresión energética de Costa Rica y servir de base para procesos de toma de decisiones requeridos para garantizar la sostenibilidad futura del sector energético en un escenario en el cual el cambio climático es un asunto crítico que compromete la trayectoria hidroeléctrica actual.

Palabras-clave: Trayectoria dependiente, transición energética, geografía económica evolutiva, energía renovable, lock-in tecnológico.

\begin{abstract}
Common research interests between geography and energy studies represent an opportunity for carrying out an in-depth examination for understanding the relationship between different trajectories of energy transition and the geographical conditions from which they emerge. By Considering Costa Rica as an empirical case study and applying theories shared between the fields of economic geography and neo-institutional theories, this research analyzes the development of Costa Rica's energy matrix by identifying three forms of path dependence mechanisms: technological lock-in; positive externalities; and institutional hysteresis. With an electricity sector almost $100 \%$ renewable and national commitments of achieving carbon neutrality, this article sheds light towards understanding a pathway that is difficult to reverse despite the issues surrounding the exploitation of conventional sources. It offers a practical example of applying economic geography theory from an evolutionary perspective in order to achieve an interpretation of Costa Rica's energy progression and serve as a basis for decision-making processes required to guarantee future sustainability of the energy sector in a scenario in which climate change is a serious issue compromising the current hydroelectricity pathway.
\end{abstract}

Keywords: path dependence, energy transition, evolutionary economic geography, renewable energy, technological lockin.

\section{INTRODUCCIÓN}


El mundialmente conocido modelo de energía renovable costarricense depende fuertemente de la hidroelectricidad, a pequeña y gran escala, un recurso que en los últimos tiempos ha estado rodeado de fuertes interrogantes ambientales y sociales. A pesar de que las fuentes de energía renovables no convencionales (geotérmicas y eólicas) fueron introducidas en el sistema de energía nacional desde el final de los años noventa, como resultado de proyectos experimentales anteriores e impulsado por las reformas de liberalización del sector, estos recursos aun juegan un papel secundario en una matriz energética en la que la energía solar, por citar un ejemplo, ha estado muy ausente, a pesar de su potencial. En contraste, la capacidad instalada de tecnologías convencionales (hidroelectricidad) ha continuado creciendo durante las últimas décadas incluso en contextos en los cuales las fuentes de energía renovable alternativas funcionarían mejor en términos de eficiencia.

Este dominio ocurre a pesar del hecho de que la generación hidroeléctrica es vulnerable a los efectos del cambio climático debido a que requiere una precipitación constante. Precisamente, Costa Rica ha tenido patrones recientes de precipitación que eventualmente podrían significar que la fuente que le dio al país su impulso de "energía verde" pueda ser restringido por los efectos del cambio climático (Fendt, 2015). Adicionalmente, existe una creciente percepción de problemas y limitaciones que surgen alrededor del desarrollo de grandes represas hidroeléctricas debido a que las mismas causan considerables degradaciones ambientales y sociales. Estos impactos han desencadenado el involucramiento y la oposición de organizaciones ambientales y de base local las cuales reclaman que las represas afectan cuencas que previamente estaban sanas al alterar la vida silvestre y desplazar comunidades indígenas.

Ante el efecto de estos eventos, se ha recurrido como fuente de respaldo a la energía fósil que es el combustible utilizado en las plantas de generación térmica. Por su parte, las alternativas sostenibles como la energía eólica, geotérmica y solar han sido relegadas dentro de la trayectoria del sistema energético nacional a pesar de que poseen fuerte potencial y ventajas en relación a su eficiencia y costo. El compromiso de Costa Rica de ser Carbono Neutral para el año 2021 elevó las expectativas para la implementación de un $100 \%$ de energías renovables dentro del sector energético al aprovechar el potencial de fuentes alternas adicionales a la hidroelectricidad, lo cual eventualmente abriría una ventana de oportunidades para promover cambios innovadores y/o radicales. Desafortunadamente, esta 
oportunidad no ha sido materializada y por lo tanto se vuelve imperativo entender el porqué, particularmente considerando que en un mundo descarbonizado se requieren nuevos enfoques para asegurar la disponibilidad y accesibilidad de servicios de energía que sean sostenibles.

Con tantos asuntos en juego y con tantas y tan fuertes implicaciones para virtualmente cada campo de las ciencias geográficas, es sorprendente la poca investigación geográfica enfocada directamente hacia asuntos energéticos. Sin embargo, las preocupaciones actuales relacionadas con la energía y el cambio climático han abierto nuevos espacios para debatir las decisiones de política energética y sus respectivas inversiones alrededor del mundo en formas que delinearán el significado y los prospectos para la sostenibilidad en sistemas humano-ambientales (Jiusto, 2009). La presente investigación considera la sostenibilidad del sistema energético de un país como un proceso en discusión en el que co-evolucionan factores políticos, económicos e institucionales con los cambios ecológicos que modifican la calidad, ubicación e impacto de los recursos energéticos.

Utilizando la teoría de path dependence (trayectorias dependientes) desarrollada dentro de la geografía económica evolutiva y la escuela de pensamiento neo-institucional, la presente investigación ofrece una explicación de la evolución energética de Costa Rica, la cual ha favorecido la generación hidroeléctrica siendo influenciada por el impacto de ciertos eventos históricos. La idea es aplicar una base teórica particular para identificar y describir esta condición, así como los mecanismos que la refuerzan y que limitan el avance de tecnologías renovables alternativas. De esta forma, se pretende ofrecer una interpretación de la matriz energética del país y servir de base para las decisiones futuras.

Como punto de partida, el presente artículo hace una breve referencia a las teorías relacionadas con las geografías de transición energética y de path dependence (trayectorias dependientes) desde la perspectiva de la geografía económica evolutiva. Seguidamente, se hace una interpretación práctica de la teoría, aludiendo al caso de la hidroelectricidad en la matriz energética de Costa Rica, mediante la explicación de los mecanismos identificados: lock-in tecnológico; externalidades positivas e histéresis institucional. A través de la aplicación de esta perspectiva teórica, que rara vez ha sido empleada en América Latina para interpretar casos prácticos, se pretende contribuir a la comprensión de la evolución 
energética en Costa Rica y a la reflexión para futuros procesos de toma de decisiones a efectos de garantizar la sostenibilidad.

\section{MARCO TEÓRICO}

\section{Geografías de transición energética}

La transición ha sido empleada analíticamente dentro de los estudios relacionados con la energía con el fin de evaluar los cambios históricos acaecidos en los diversos sistemas energéticos a escala nacional y global (Smil, 2010). Las transiciones energéticas han sido asociadas históricamente con cambios sociales extensos, incluyendo los procesos de industrialización y urbanización dentro de una sociedad predominantemente consumista. El aseguramiento de la generación y distribución de la energía en una sociedad tiene una influencia poderosa en la prosperidad económica, la estructura geográfica y las relaciones internacionales; aspectos que están directamente relacionados con la dimensión territorial de la transición energética (Bridge et al, 2013).

Los sistemas de infraestructura relacionados con la generación, transmisión y distribución energética están constituidos especialmente y han sido territorializados de diversas maneras a lo largo del tiempo. En este sentido, la territorialización del proceso de transición energética se evidencia de dos maneras. Por un lado, según la distribución de diversas actividades relacionadas con la energía a través de un determinado espacio y los procesos causales que hacen surgir dichas configuraciones; y por otro lado, por las conexiones e interacciones geográficas entre ese espacio y otros espacios (p. ej. la posición de Costa Rica en un ámbito más amplio de política económica de estados, firmas multinacionales, acuerdos internacionales y ONGs) (García \& Avendaño, 2018).

El estudio de la innovación estructural dentro de los sistemas de transición energética considera metáforas geográficas tales como nicho, régimen y paisaje a efectos de proveer una interpretación contextualizada del cambio tecnológico y entender las innovaciones del sistema en el tiempo (Grin, 2012). Si bien esta aproximación ha contribuido al entendimiento sobre cómo ocurre la transición, las preocupaciones primarias se refieren al desenvolvimiento de un proceso temporal y a la identificación de 
factores que causan que algunos nichos evolucionen o sean incorporados en regímenes y otros no. Estas preocupaciones han tendido a circunscribir cualquier atención formal al espacio, lugar y escala geográfica dentro de las teorías de transición convencionales (Bridge, Bouzarovski, Bradshaw, \& Eyre, 2013).

En este sentido, examinar la transición energética como un proceso geográfico comprende prestarle atención a la reconfiguración de los patrones actuales y a las escalas de actividad económica y social; lo anterior se debe a que los sistemas de energía están espacialmente constituidos y por eso su transición requiere interpretación geográfica considerando que sus componentes están insertos en escenarios particulares y la red resultante produce geografías de conexión, dependencia y control (Emmerson \& Stevens, 2012). Por ejemplo, la generación de electricidad baja en carbono puede ser alcanzada por medio de actores grandes y remotos (tales como la hidroelectricidad en el caso de Costa Rica) y transmisiones de larga distancia, vía mini redes o por medio de micro generación altamente descentralizada. De igual manera, los diferentes elementos de una política para promover la seguridad energética, desde la inversión doméstica y el proceso de toma de decisiones hasta controlar y proteger el abastecimiento de energía, se basan en presunciones sobre la escala geográfica desde la cual los sistemas de energía deberían estar gobernados.

La geografía también representa tierra fértil para estudiar el proceso de transición energética considerando que las contradicciones socio ecológicas son cada vez más evidentes en los sistemas de energía convencionales que se basan en una demanda exponencialmente creciente atendido primordialmente por medio de la expansión de suministros de combustibles fósiles y, como en el caso de Costa Rica, por medio de extensos proyectos hidroeléctricos. Con tantos asuntos en juego e implicaciones fuertes para casi todas las áreas de las ciencias geográficas, sorprende lo poco que se enfoca la investigación geográfica directamente en asuntos energéticos.

Esta poca disposición puede reflejar un desequilibrio entre los métodos heterogéneos, sociales y teóricamente informados y las preocupaciones de la geografía y la frecuente naturaleza técnica y económica de la investigación de las ciencias sociales acerca de la energía. Las preocupaciones sobre el cambio climático son, de cierto modo, una oportunidad para moldear el significado y los prospectos para 
la sostenibilidad en sistemas humano- ambientales (Jiusto, 2009). Cumplir con los retos del cambio climático y la seguridad energética es, por lo tanto, un proyecto fundamentalmente geográfico: no solo requiere que las sociedades se comprometan a inversiones masivas para rediseñar la infraestructura, los edificios, los equipos y aparatos, sino también a ampliar las posibilidades de elección entre una variedad de posibles soluciones espaciales y escalas de gobernanza.

No obstante, la investigación en el campo de la transición energética ha brindado poca atención a los procesos históricos dentro del panorama económico y no ha ofrecido una interpretación sobre cómo dicho panorama evoluciona en el tiempo. En este sentido, hay un alto potencial para poder lograr una comprensión más integral de asuntos como la geografía y su relación con el progreso tecnológico, las ventajas competitivas dinámicas, la reestructuración económica y el crecimiento económico (Boschma \& Martin, 2012). Este contexto ofrece la posibilidad de aplicar y extender las ideas y conceptos de economías evolutivas dentro del análisis y la comprensión de los procesos de toma de decisiones asociadas con la transición de la energía.

\section{Geografía económica evolutiva y las teorías de path dependence}

En las últimas tres décadas se han realizado contribuciones teóricas importantes en el campo de la geografía económica. Este campo de investigación se ha apartado del análisis económico tradicional para buscar diversas contribuciones de la economía heterodoxa y de otras ciencias sociales ajenas a la rama de la economía, incluyendo los fundamentos institucionales, culturales y sociales del desarrollo urbano y cultural. (Martin \& Sunley, 2007) (Martin, 2008) (Boschma \& Martin, 2010). Al mismo tiempo, varios economistas liderados por Paul Krugman y Michael Porter han resaltado la importancia de una perspectiva geográfica en el entendimiento de la dinámica y competitividad de la economía.

Por su parte, la economía evolutiva se enfoca en los procesos y mecanismos por medio de los cuales la economía se auto transforma a sí misma desde adentro por medio de procesos históricos de cambio y desarrollo (Witt, 2003). Ésta discute cómo los actores y las instituciones han desarrollado rutinas y hábitos duraderos, alcanzando un carácter cuasi genético, con el funcionamiento del mercado como un 
importante mecanismo de selección (MacKinnon et al., 2009). El propósito de emplear tales conceptos, teorías y metodologías dentro de la geografía económica es para utilizarlos en la interpretación y explicación de los cambios en el panorama histórico.

Este amalgama es útil para demostrar cómo el entendimiento de los procesos que maneja la evolución económica mejora situando a la economía en el espacio, demostrando la relevancia de la geografía en la determinación de la naturaleza y trayectoria de la evolución del sistema económico. Una de las ideas más interesantes en la geografía económica evolutiva es que la historia de una industria (p. ej. la transición energética) literalmente se encuentra en el presente, lo cual significa que las decisiones tomadas en el pasado influyen las decisiones subsecuentes de dicha industria en cuanto a su método, diseño y prácticas.

Dentro de la geografía económica evolutiva es posible distinguir tres enfoques principales al estudio de índole evolutivo: el Darwinismo generalizado, la teoría de los sistemas complejos adaptativos y la teoría de path dependence. Esta tercera aproximación, considerada en la discusión de la presente investigación, se ocupa de enfatizar la dimensión histórica de la economía. Los procesos de path dependence y lock-in son dependientes del lugar y como tales requieren explicación geográfica (Martin \& Sunley, 2006).

La discusión de la teoría de path dependence dentro de la geografía económica evolutiva es un resultado de la integración de la economía evolutiva y la investigación espacial. Enfatiza la importancia del desarrollo económico histórico a largo plazo (Lengyel \& Molnar, 2015) enfocándose en procesos en los cuales la situación actual depende de su propia historia, aunque no al extremo de que la misma eventualmente regrese a su estado previo. Se caracteriza por un grado de irreversibilidad y mecanismos auto reafirmantes (Martin \& Sunley, 2010). De ahí proviene el concepto de lock-in (bloqueo o aseguramiento), en otras palabras, la intención es que el concepto capture la forma en la cual eventos históricos pequeños y contingentes pueden activar mecanismos y procesos auto reafirmantes que "encierran" estructuras y trayectorias de desarrollo particulares. Las estructuras y trayectorias que resulten "encerradas" o "bloqueadas" dependerá de la secuencia particular de eventos que se van desarrollando paulatinamente (Martin \& Sunley, 2006). 
Como desventaja se señala que desde la geografía económica se ha aplicado erróneamente el concepto de path dependence como si el mismo fuese obvio y absolutamente incontestable Es importante resaltar el hecho de que detrás de la noción de path dependence existen una serie de factores que en conjunto suman hacia una preferencia direccional. Los factores que la causan difícilmente son comunicados, lo que frecuentemente conduce de manera equivocada a una invocación unidimensional del término. Para evitar esta mala interpretación teórica, la literatura sobre geografía económica evolutiva retrata tres formas de mecanismos de path dependence.

Primero, path dependence se refiere a lock-in tecnológicos cuando una trayectoria específica resulta de una serie histórica de acciones de las cuales la desviación no es posible a pesar de la oportunidad que ofrece implementar tecnologías alternativas y posiblemente más eficientes. Segundo, los efectos de aglomeración y las externalidades positivas pueden reforzar el path dependence conforme los ingresos crecientes generan respuestas positivas que consolidan trayectorias de desarrollo que fueron previamente establecidas. Tercero, la progresiva naturaleza auto reproductiva de instituciones formales e informales, estructuras sociales y rasgos culturales conocidos como histéresis, también influyen en las trayectorias dependientes mediante la estimulación de la estabilización, institucionalización e inserción social de ciertas actividades (Martin \& Sunley, 2010).

La presente es una investigación empírica que considera el caso del sector energético de Costa Rica al que se le aplica un análisis histórico, geográfico y económico, cuyos resultados se pueden explicar a través de la teoría en forma de un lock-in tecnológico, de infraestructura, institucional y conductual. Mientras los gobiernos invierten en grandes proyectos de para proveer acceso a energía barata a efectos de que se estimule el desarrollo económico y la reducción de la pobreza, se arriesgan a que sus economías se hagan dependientes de trayectorias energético-intensivas que son difíciles de revertir (Fouquet, 2016). En países como Costa Rica, donde predomina la energía hidroeléctrica y la industria está concentrada y puede ser influenciada por decisiones políticas, un cambio hacia sistemas energéticos más innovadores y eficientes se torna aún más difícil (García, 2014).

\section{La energía hidroeléctrica en la matriz energética de Costa Rica}


Costa Rica ha sido catalogada como uno de los mayores usuarios de energía renovable en el mundo y durante cierto tiempo ha generado el 100\% con energía renovable. Durante el 2018, el suministro de electricidad del país fue generado por hidroelectricidad (74\%), eólico (16\%), geotérmico (8\%), combustibles fósiles $(>1 \%)$ y biomasa/solar ( $<1 \%)$ (ARESEP, 2018). De esta manera, las fuentes renovables abastecieron casi el 99\% de las necesidades energéticas del país, aun y cuando se basa intensamente en plantas hidroeléctricas que realizan la mayoría de la generación. Si bien el país cuenta con gran potencial para generar energía solar y a partir de biomasa, no saca ventaja de esta condición debido a que únicamente un $0.01 \%$ de la energía consumida proviene del sol y continúa representando un porcentaje prácticamente insignificante dentro de la matriz energética.

Aunque la energía hidroeléctrica representa las 3\%4 partes de la generación eléctrica del país, el caso costarricense de uso de energías renovables es notable porque no depende completamente de la hidroelectricidad. Esta condición difiere de otras naciones en vías de desarrollo con recursos limitados para invertir en fuentes renovables y mercados de energía pequeños o con alto potencial hidroeléctrico. Por otro lado, aunque el país posee reservas comprobadas de petróleo y gas natural, se ha rehusado a explotar este tipo de inversiones debido a los impactos asociados (Wilde-Ramsing \& Potter, 2008).

También es excepcional que el sector de generación y distribución de electricidad sea dirigido por una empresa estatal - Instituto Costarricense de Electricidad (ICE) -. No obstante, actores privados también participan en la generación de electricidad a partir de las reformas liberales implementadas en el sector durante los años 90. Esta configuración institucional ha permitido un mayor control sobre la asignación de recursos ambientales y desarrollo económico.

Sin embargo, varios actores han cuestionado este modelo de energía renovable, especialmente en años recientes. Por un lado, a pesar de la relativa estabilidad económica y la ambición de convertirse en un país carbono neutral para el 2021, fuera del subsector eléctrico, el sector transporte continua dependiendo de la importación de combustibles fósiles. Por otro lado, la producción de energía renovable se encuentra en una encrucijada debido a su dependencia en la hidroelectricidad, lo cual ha provocado que ante la variación de las precipitaciones y los episodios de sequías se haya incrementado el uso de 
combustibles fósiles para asegurar el suministro de energía a corto plazo (García, 2014; Wilde-Ramsing \& Potter, 2008).

\section{Mecanismos de lock-in y path dependence}

Desde una perspectiva teórica, la noción de path dependence es rara vez unidimensional. Igualmente, desde un punto de vista empírico, la decisión de generar energía por medio de una combinación de diferentes recursos tiene implicaciones sobre varios factores. Por un lado, es importante debido a factores económicos (p. ej. el incremento en la importación de combustibles fósiles), ambientales (p. ej. conservación de la naturaleza o en términos de emisión de dióxido de carbono) y sociales (p. ej. accesibilidad a la electricidad). Por otro lado, también por razones políticas, ya que estas decisiones ponen en evidencia los intereses de los actores involucrados, además de repercutir sobre la reputación internacional del país como modelo de energía renovable o país carbono neutro.

Desde un punto de vista aplicado, esta investigación tiene como objetivo mejorar la comprensión sobre la toma de decisiones dentro del sector eléctrico de Costa Rica. Para ello se explican a continuación tres formas o mecanismos de path dependence identificados: a) el lock-in o bloqueo tecnológico resultante de una serie de procesos históricos a partir de los cuales es difícil el cambio; b) las externalidades positivas que refuerzan el path dependence y que consolidan el desarrollo de las trayectorias que fueron previamente establecidas; y, c) la histéresis institucional que promueve la estabilización e inserción social de ciertas actividades. Estos mecanismos se activan en diferentes etapas históricas de la transición energética de Costa Rica según se explica a continuación. 


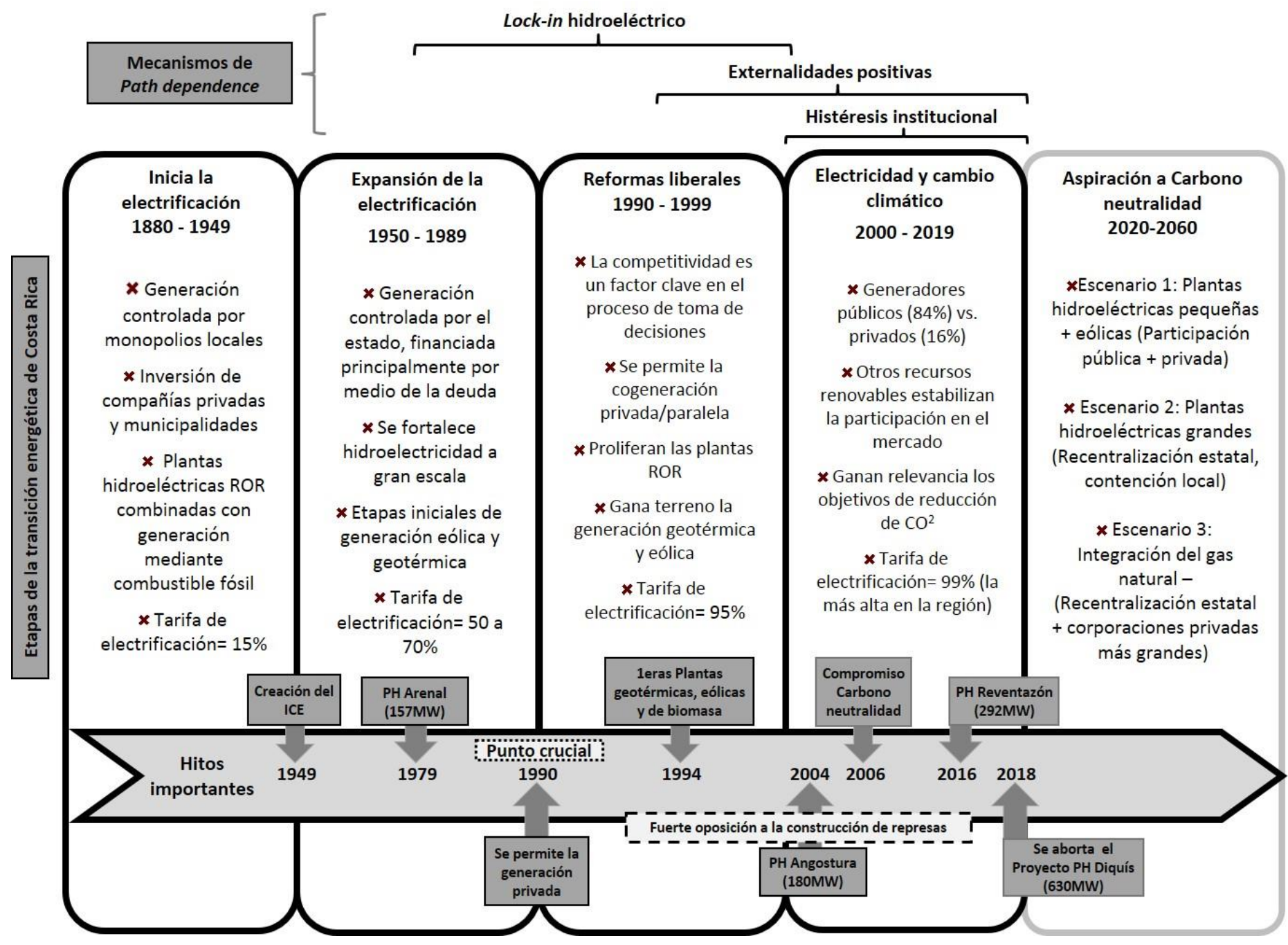

Figura 1. Mecanismos de lock-in y path dependence dentro de la evolución energética de Costa Rica

(Fuente: investigación propia) 


\section{El lock-in de la hidroelectricidad}

La serie de acciones históricas que resultan en el lock-in tecnológico tienen su origen en el período de la expansión de la electricidad (1950-1989). La Guerra Civil de 1948 dio paso a la era del "desarrollo" en la cual el estado jugó un rol preponderante en la economía y bienestar del país, incluyendo al sector eléctrico (Bull, 2005). El Instituto Costarricense de Electricidad (ICE) fue creado en 1949 como una institución pública descentralizada, verticalmente integrada que aún hoy es la principal empresa de energía en Costa Rica.

El aumento de la cobertura eléctrica, las tarifas eléctricas con orientación social, así como el desarrollo y la mejora constante de la red de electrificación, fueron logros del ICE muy importantes para el progreso del Estado durante esta época. Otras compañías (p. ej. Distribuidoras de electricidad municipales y cooperativas) también fueron incluidas en el modelo junto al ICE a efectos de electrificar los territorios rurales y aislados del país. En este contexto, la generación de electricidad por medio de plantas hidroeléctricas ofrecía ventajas económicas para asegurar la energía necesaria a la industria doméstica y el desarrollo continuo de la red eléctrica (Vargas, 2002).

Considerando que el agua era un recurso abundante en el país, la energía hidroeléctrica se convirtió en una fuente extremadamente importante por dos razones principales: primero, al garantizar el suministro de energía para la demanda doméstica industrial y la expansión eléctrica; y, segundo, para mantener tarifas socialmente orientadas en favor de consumidores residenciales, áreas rurales y algunos sectores industriales (García, 2012). Las plantas generadoras alimentadas mediante combustible fósil se incorporaron a la red nacional desde un comienzo, particularmente como plantas de respaldo durante el pico de la demanda, aunque prácticamente no fueron necesarias hasta la década de los 90 (WildeRamsing \& Potter, 2008). Al final del período de expansión de la electrificación, la energía solar se encontraba en una etapa experimental, la alternativa geotérmica estaba aún en su etapa de prefactibilidad y los estudios preliminares para la ubicación de las plantas eólicas apenas estaban en ejecución. Sin embargo, el suministro de grandes cantidades de energía a bajo costo evolucionó lo suficiente para crear un lock-in tecnológico de la generación hidroeléctrica que perdura en el tiempo. 
Según García (2014), las reformas liberales durante los años noventa fueron un punto crucial en la trayectoria energética nacional porque desencadenaron una serie de transformaciones que aunque limitadas, tuvieron impactos significativos sobre la naturaleza de la producción y la incorporación de nuevas fuentes de energía. Entre las reformas cabe citar la incorporación de la participación del sector privado, o cogeneración, y un sistema tarifario que creaba competencia entre los productores de electricidad, lo que en consecuencia limitaba las finanzas del ICE (Merino, 2003). La generación privada de electricidad y su correspondiente cuota de participación en el mercado energético estaban y continúan siendo limitadas por ley (Ley $\mathrm{N}^{\circ} 7200$ de 1990 y Ley $\mathrm{N}^{\circ} 7508$ de 1995).

Como resultado, un 14\% de la capacidad instalada pasó a manos de productores privados. En términos de plantas eléctricas, si bien se incorporaron nuevas fuentes de energía como la eólica, la hidroeléctrica siguió siendo la más utilizada por los nuevos actores privados. De hecho, la incorporación de inversionistas privados dio paso al desarrollo de varias plantas hidroeléctricas que aprovechan directamente la escorrentía del río (a filo de agua) sin la necesidad de construir un gran embalse. Las primeras plantas de energía geotérmica y eólica aparecieron durante este periodo, sin embargo, con una participación limitada en la matriz energética del país. Algunas de estas nuevas plantas estaban en manos de ICE y otras de empresas privadas, sin embargo cabe destacar que los avances en estas tecnologías fueron apoyados en sus etapas iniciales por el ICE y sus socios internacionales que hicieron posible la transferencia tecnológica.

En términos de relación estado-sociedad, este periodo desencadenó nuevos acuerdos entre los actores del sector eléctrico, pero también conflictos latentes en términos de relaciones entre estado, actores privados y organizaciones comunales de base. Primero, la aprobación de las reformas liberales resultaron en pugnas políticas con fuerte oposición por parte de un amplio sector de los ciudadanos del país que se oponían a la privatización del sector (Hoffman, 2007). Segundo, la competencia de nuevos actores sobre el recurso hídrico motivó el surgimiento de conflictos socio-ambientales especialmente en las zonas rurales (Sanchez et al., 2010). 
Así por ejemplo, durante 1996 varias comunidades de la Zona Sur crearon el primer Comité de Defensa de los Ríos en contra del proyecto hidroeléctrico privado Los Gemelos, y en defensa de la protección de la cuenca del Rio Chirripó Pacífico (Municipalidad de Pérez Zeledón, 2013). Las movilizaciones ambientales también emergieron en otras regiones del país, oponiéndose al desarrollo de proyectos hidroeléctricos, tanto públicos como privados (p. ej. a los proyectos en el río Pacuare en el Caribe y en el río Savegre en el Pacífico Central) (Sanchez et al., 2010; FECON, 2014).

Mientras tanto, este periodo fue testigo de cómo el ICE continuó con la ejecución de grandes plantas hidroeléctricas. Durante los años 90 se concluyó el gran complejo hidroeléctrico Arenal (157MW), posteriormente inició operaciones el complejo hidroeléctrico Toro Amarillo con 5 años de retraso en su construcción debido a litigios relacionados con expropiaciones. En la década siguiente, el ICE inauguró el proyecto hidroeléctrico Angostura (180MW), la planta más grande de ese periodo.

De acuerdo a los planes de expansión, el siguiente proyecto grande habría sido la megarepresa de Boruca (1500MW) en la Zona Sur y que se previó sería inaugurado en el 2010; este proyecto luego se redujo y se renombró como Diquís (630MW). Sin embargo, estos grandes proyectos fueron eventualmente descartados al verse envueltos en una serie de largos procesos de resolución de conflictos, principalmente debido a que habrían inundado porciones de territorios indígenas y ecosistemas de humedales protegidos (Carls \& Haffar, 2010).

El desarrollo continuo de grandes represas por parte del ICE se justificó con el propósito de apoyar el crecimiento económico nacional, pero además para expandir las ventas de electricidad fuera del país. La ratificación del Protocolo del Mercado Eléctrico Centroamericano proporcionó incentivos para lograr una demanda regional de grandes cantidades de electricidad a bajo costo. En este sentido, las grandes represas hidroeléctricas y las pequeñas plantas a filo de agua se consideraron adecuadas para este propósito. Por consiguiente, la hidroelectricidad siguió siendo la fuente principal tanto para el sector público como privado, mientras que la energía geotérmica y la eólica se convirtieron en parte del sistema de energía, pero con participación limitada. 


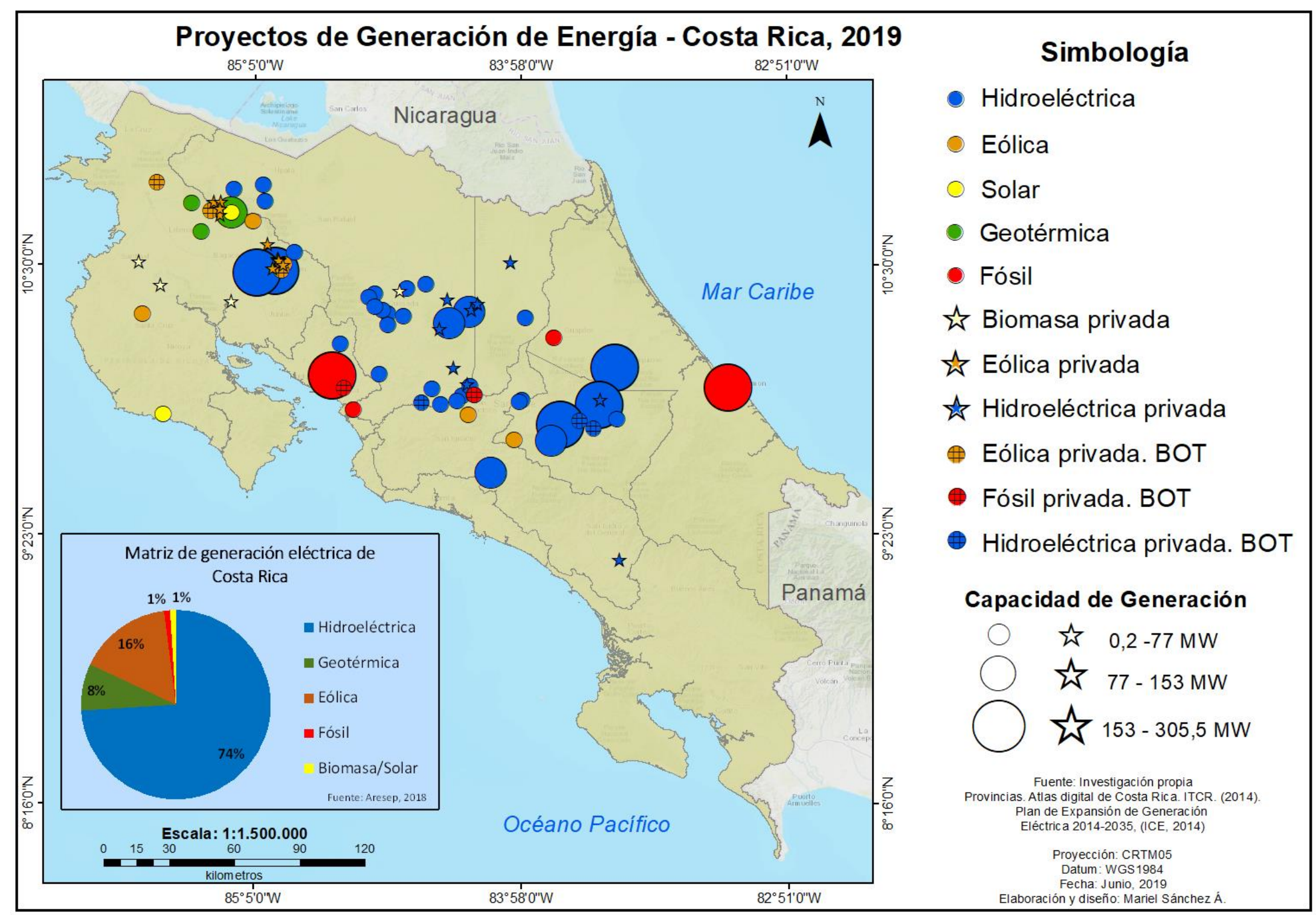

Figura 2. Proyectos de Generacíon de Enérgia - Costa Rica, 2019 


\section{Externalidades positivas que refuerzan el path dependence}

Las reformas liberales anteriormente discutidas son consideradas decisivas, ya que desencadenaron en una serie de mecanismos que refuerzan el lock-in tecnológico de la hidroelectricidad en Costa Rica. Desde entonces, dicho patrón de producción ha sido reproducido, creando efectos de aglomeración (p. ej. al incrementar el número de plantas hidroeléctricas a filo de agua), activando las externalidades positivas por medio de políticas que incorporan nuevos actores (p. ej. inversionistas privados) y generando beneficios privados a través de la generación de hidroelectricidad.

Adicionalmente, se activaron los mecanismos de coordinación, los cuales operan a través de las ganancias en eficiencia a partir de una regla o norma que hacen que las conductas puedan ser anticipadas. De esta forma, los efectos de coordinación hacen atractivo para otros adoptar y seguir la misma regla, ley o norma (p. ej. objetivos, límites y estándares). Así por ejemplo, desde los inicios del desarrollo hidroeléctrico del país, diferentes actores adoptaron y aplicaron leyes y normas para desarrollar el potencial de este recurso con el objetivo de alcanzar el bienestar social y económico a escala nacional. Para el sector privado y la futura orientación institucional del sector, las reformas liberales incrementaron sus competencias y dieron pie a las externalidades positivas explicadas.

Posterior a las reformas liberales, los efectos de coordinación también alteraron las decisiones individuales para la producción de electricidad por medio de tres normas regulatorias que pueden ser agrupadas en: regulaciones de mercado, requerimientos de evaluación de impacto ambiental, e incentivos para la mitigación de gases de efecto invernadero (p.ej. certificados de carbono). Estas normas empezaron a operar casi simultáneamente y comenzaron a alterar las decisiones sobre energía después de los años 90 .

Evidencia de estos efectos de coordinación brindan una posible explicación del predominio de proyectos hidroeléctricos, el avance de los parques eólicos y geotérmicos, así como los pasos iniciales hacia la generación de energía solar a mayor escala. Otros mecanismos que se pueden identifican son los efectos del aprendizaje. A través de éstos, se gana eficiencia al ejecutar repeticiones sucesivas de una tarea, lo 
que permite generar destrezas que luego repercuten en mejores resultados. Por ende, el tiempo es una condición necesaria para desarrollar estos efectos.

A lo largo de la historia de la hidroelectricidad en el país, desde la primera planta hidroeléctrica construida en 1880, y con más de medio siglo bajo el control estatal del ICE, se fueron desarrollando la expertise tecnológica y las destrezas en su aplicación de forma satisfactoria. En este sentido, los técnicos del ICE adquirieron destrezas y ganaron experiencia en la construcción de plantas hidroeléctricas, las cuales crecieron con el tiempo, no solo en términos de conocimiento y habilidades, sino también en términos del tamaño y escala de las instalaciones construidas. Estos efectos del aprendizaje no sólo condujeron a estos resultados en el caso del ICE y otras compañías estatales como las cooperativas de producción eléctrica, sino también fueron relevantes para el sector privado. Desde este punto de vista, las reformas de liberalización del sector dieron paso a la construcción de varias centrales hidroeléctricas a pequeña escala, tipo filo de agua, financiadas precisamente por inversionistas privados, muchos ellos exempleados del ICE con experiencia previa en este tipo de obras.

A pesar de la oportunidad que se abrió para aumentar la contribución en la matriz energética nacional de las tecnologías renovables alternativas, con un mejor desempeño que la hidroeléctrica convencional en términos de reducción del riesgo asociado con el cambio climático, el impacto en los ecosistemas, las reservas acuíferas y las comunidades indígenas, esta no se aprovechó. El lock-in hidroeléctrico persistió y hasta la fecha no se percibe una desviación de dicho patrón en un futuro cercano. Los mecanismos que operan en la reproducción de este resultado a través del tiempo incluyen economías de escala, los efectos del aprendizaje y los efectos de coordinación explicados anteriormente. Aunque menos explorados, otros mecanismos relacionados con intereses creados e institucionalizados dentro del sector energético también sostuvieron el desarrollo de la hidroelectricidad. Todos ellos producen externalidades positivas que explican el path dependence en la hidroelectricidad.

\section{Histéresis institucional e inserción social}


Desde sus orígenes y durante la "era de desarrollo estatal", el ICE exhibió características de un monopolio natural en términos de generación de energía. Las represas hidroeléctricas brindaron la infraestructura para lograr los objetivos estatales de suministro eléctrico para la industria doméstica y para expandir la red de conexiones (Vargas, 2009). Durante este periodo los empleados del ICE adquirieron experiencia tecnológica y eficiencia en la construcción de instalaciones hidroeléctricas.

El path dependence de la hidroelectricidad en Costa Rica se ha fortalecido por medio de la histéresis institucional del ICE, significando esto que existe una progresiva naturaleza auto reproductiva de esta organización dentro de la estructura social del país e incluso con rasgos culturales. Esto se traduce, en el ámbito de la geografía económica evolutiva, como un caso de inercia institucional que causa estabilización, persistencia y pérdida de flexibilidad institucional, fortaleciendo la progresiva naturaleza auto reproductiva de instituciones que favorecen la generación de hidroelectricidad sobre otras fuentes renovables alternativas.

En suma, la hidroelectricidad ha sido más que una fuente de energía en Costa Rica, al mismo tiempo que el ICE ha sido más que un simple proveedor de electricidad. El ICE ha alcanzado buena reputación y credibilidad en relación a su eficiencia y estándares técnicos. La autonomía concedida a esta entidad en su momento no solo fortaleció su liderazgo en el sector energético, sino también dio paso a un actor político con participación significativa en la formulación de políticas futuras. Durante el inicio del siglo XXI dicho instituto era considerado como "el último refugio de propiedad estatal en América Latina" (Bull, 2005, p. 82).

Además, Costa Rica fue el primer país en unirse a países industrializados en el compromiso de compensar sus emisiones de gases de efecto invernadero (GHG) (Landreau, 2006). El país también fue pionero en el desarrollo del Pago por Servicios Ambientales (PSA), vendiendo la reducción de emisiones a través de la captura del carbono del bosque por medio de Certificados de Compensación (CTO por sus siglas en inglés) conforme al Protocolo de Kyoto. En este caso, los efectos de coordinación hicieron que la creación de un mercado global de carbono fuera una regla o norma atractiva de seguir para actores privados y 
estatales a nivel nacional e internacional. Estas nuevas instituciones o normas ayudaron a fortalecer la trayectoria hidroeléctrica.

Esta condición se refuerza en el contexto actual, ya que el país aspira a tener acceso y convertirse en miembro de la Organización para la Cooperación y el Desarrollo Económico (OCDE). Además es fundamental tener claro que la energía hidroeléctrica es una tecnología clave dentro de la matriz de energía renovable necesaria para la des-carbonización de la economía del país.

A pesar de que el país ganó reconocimiento internacional por la implementación exitosa de políticas alineadas con la estrategia del cambio climático global basada en la compensación de emisiones y en la actualidad con su compromiso de alcanzar la carbono neutralidad al 2021, la diversificación en el uso de fuentes de generación renovables no es considerada urgente debido a que la trayectoria institucional actual basada en hidroelectricidad ya de por sí es baja en emisiones de carbono.

Al ser una tecnología establecida favorecida por el ICE para la generación de electricidad en Costa Rica, la energía hidroeléctrica domina el sistema energético nacional y no se espera que disminuya en los años siguientes. Además, su desarrollo actual se torna predecible o incluso irreversible dada la estructura institucional y la planeación del sector eléctrico del país. Aun cuando las tecnologías renovables alternativas podrían tener una relevancia mayor en el sistema energético nacional, las instituciones energéticas establecidas y de larga data, restringen su incorporación activa.

\section{CONCLUSIONES}

En el contexto Latino Americano rara vez han sido explorados el cambio institucional y las dinámicas institucionales bajo el enfoque de path dependence. La presente investigación plantea que para un abordaje evolutivo en la geografía económica existe una necesidad de reconocer y conceptualizar la evolución institucional y sus cambios con mayor profundidad. Es necesario convertirlos en parte integral del análisis y explicar cómo las instituciones, entendidas como leyes, normas y reglas, juegan un rol en 
el desarrollo dinámico de las trayectorias evolutivas, especialmente para el entendimiento de dinámicas económicas de largo plazo en espacio y tiempo.

Este artículo considera las disposiciones institucionales de la transición energética de Costa Rica como caso de estudio del path dependence. El país se considera líder en el uso de energía renovable debido a que más del $90 \%$ de su electricidad ha sido producida durante décadas con fuentes renovables, principalmente hidroelectricidad. Las fuentes renovables alternativas, tales como la geotérmica y eólica, fueron introducidas en el sistema nacional energético como resultados de varios proyectos experimentales desde los años 70's y posteriormente introducidos después de las reformas de liberalización del sector durante fines de los 90's.

Sin embargo, más de tres décadas después de estos episodios, las tecnologías renovables alternativas como la energía solar o biomasa permanecen marginadas, mientras la energía hidroeléctrica se reproduce a pesar de sus limitaciones en un contexto distinto. Esta trayectoria bajo el dominio de la energía hidroeléctrica tiene sus propios procesos que la favorecen y refuerzan, al mismo tiempo que dificultan el avance de otras tecnologías renovables. Interpretar estos procesos mediante la identificación de los actores participantes y los hechos ocurridos es una parte importante del abordaje analítico utilizado en esta investigación para la determinación de un resultado tecnológico y además político.

El análisis de la trayectoria eléctrica del país, conducida por eventos históricos y reproducida por los efectos de ciertos mecanismos, brindan evidencia que refuerza la idea de presentar un caso de path dependence en la hidroelectricidad. Primero, el presente análisis identificó un lock-in tecnológico en el sector eléctrico de Costa Rica. Considerando las condiciones geográficas del país (p. ej. topografía y precipitación), desde sus orígenes el ICE se enfocó en el desarrollo de la hidroelectricidad como una fuente que garantizó economías de escala al suministrar grandes cantidades de electricidad a precios razonablemente bajos, lo cual fue crucial para promover el desarrollo y el bienestar.

Un punto crucial en este desarrollo fueron las políticas de liberalización económica aprobadas durante los años noventa que no sólo fomentaron la participación del sector privado en el mercado de la 
generación de electricidad y el algunos proyectos con energías renovables alternativas, sino que también aumentaron la producción de energía hidroeléctrica. Desde entonces, esta condición de lock-in hidroeléctrico ha sido reforzada por las políticas públicas y nuevas demandas de energía que han evolucionado en una trayectoria difícil de revertir y que, además, ha desaprovechado la oportunidad de obtener mayores contribuciones de tecnologías renovables alternativas.

Segundo, las externalidades positivas que han reforzado la dependencia del país en la generación hidroeléctrica fueron motivadas por dos razones principales. Por un lado, la necesidad de garantizar el suministro de energía para la demanda doméstica industrial, continuar el avance de la electrificación en todo el país y el mantenimiento de tarifas socialmente orientadas a los consumidores residenciales, industriales y rurales. Por otro lado, las reformas liberales de los 90 que abrieron el mercado eléctrico a la generación privada también favorecieron la hidroelectricidad. De manera que las decisiones que se tomaron para desarrollar proyectos hidroeléctricos, públicos o privados, fueron económicas, sociales y políticas y los mecanismos que desencadenaron las externalidades positivas fueron: economías de escala, efectos de aglomeración, efectos de coordinación y efectos de aprendizaje.

La tercera forma de mecanismos de path dependence es la histéresis institucional e integración social relacionados con la creación del ICE como una entidad que no sólo es el líder del sector energético del país, sino también un poderoso actor político en la conformación de las condiciones de bienestar y desarrollo social del país. Sin embargo, desde un punto de vista de diversificación de la matriz energética, el ICE experimenta una inercia institucional, motivada principalmente por los mecanismos anteriores.

Asegurar la generación de energía y transformarla con fines útiles influye en la prosperidad económica, la estructura geográfica y las relaciones internacionales de la sociedad. En el mundo actual con aspiraciones de un desarrollo bajo en carbono, se necesitarán nuevos enfoques y nuevas geografías para garantizar la disponibilidad y accesibilidad de los servicios energéticos sostenibles. La presente interpretación de la transición energética de Costa Rica y su posible path dependence hidroeléctrico, ofrece una mirada para abordar la transición hacia sistemas de energía más sostenibles y diversos, sin 
perder de vista los grandes logros históricos del desarrollo eléctrico que garanticen el acceso universal a servicios eléctricos seguros, eficientes y bajos en carbono.

\section{REFERÊNCIAS}

ARESEP. (2018). Autoridad Reguladora de los Servicios Públicos (ARESEP): Estadísticas del Mercado Eléctrico Nacional. Retrieved June 26, 2019, from

https://aresep.go.cr/electricidad/index.php?option=com_content\&view=article\&id=1389\&catid=106

Boschma, R., \& Martin, R. (2010). The aims and scope of evolutionary economic geography. En R. Boschma, \& R. Martin (2012), The Handbook of Evolutionary Economic Geography (págs. 3-39). Cheltenham, U.K.: Edward Elgar Publishing.

Boschma, R., \& Martin, R. (2012). The Handbook of Evolutionary Economic Geography. (R. Boschma, \& R. Martin, Edits.) Cheltenham, U.K.: Edward Elgar Publishing.

Bridge, G., Bouzarovski, S., Bradshaw, M., \& Eyre, N. (2013). Geographies of energy transition: Space, place and the lowcarbon economy. Energy Policy, 53, 331-340.

Bull, B. (2005). Aid, power and privatization: The politics of telecommunication reform in Central America. Cheltenham, U.K.: Edward Elga Publishing.

Carls, J., \& Haffar, W. (2010). Conflict Resolution of the Boruca Hydro-Energy Project: Renewable Energy Production in Costa Rica. New York: Continuum.

Emmerson, C., \& Stevens, P. (2012). Maritime Choke Points and the Global Energy System: Charting a Way Forward. Chatham House Briefing Paper. London, U.K.: Chatham House (the Royal Institute of International Affairs).

FECON. (2014). Mesa de diálogo nacional sobre energía eléctrica: Pronunciamiento y propuestas de las comunidades del Pacuare-Duchí y Savegre-Acosta. Obtenido de: http://www.feconcr.org/doc/dialogoelectrico/Pacuaresavegre.pdf

Fendt, L. (30 de March de 2015). The truth behind Costa Rica's renewable energy. Obtenido de The Guardian: https://www.theguardian.com/commentisfree/2015/mar/30/truth-behind-costa-rica-renewable-energy-reservoirs-climatechange

Fouquet, R. (2016). Path dependence in energy systems and economic development. Nature Energy, 1. Obtenido de http://eprints.lse.ac.uk/67119/1/Fouquet_Path\%20dependence_2016.pdf

García, D. (2012). Decision-making and the energy path towards carbon neutrality: the case of Costa Rica. Berlin Conference on Evidence for Sustainable Development. Berlin, Deutschland: Environmental Policy Research Centre (FFU) Freie Universität Berlin

García, D. (2014). Solar energy and the problem of path dependence in Costa Rica's energy system. Hamburg, Deutschland: Dissertation zur Erlangung der Würde einer Doktorin der Wirtschafts und Sozialwissenschaften, Fakultät Wirtschaft und Sozialwissenschaften, Universität Hamburg.

García, D., \& Avendaño, D. (2018). Energy transition and path dependence: The case of Costa Rica. Revista Geográfica de América Central, 61E (3), 281-296.

Grin, J. (2012). The governance of transitions and its politics. Conceptual lessons from the earlier agricultural transition and implications for transition management. International Journal of Sustainable Development, 72-89.

Hoffman, B. (2007). Why reforms fail: The politics of policies in Costa Rican telecommunications liberalizations (Vol. 47). Hamburg, Deutschland: GIGA Working Papers.

Jiusto, S. (2009). Energy transformations and geographic research. En N. Castree, D. Demeritt, D. Liverman, \& B. Rhoads, A Companion to Environmental Geography (págs. 533-551). Oxford, UK: Blackwell Publishing Ltd. 
Landreau, B. (2006). Evaluación del Mecanismo de Desarrollo Limpio en Costa Rica. (Tésis de maestría en gestión ambiental y ecoturismo con énfasis en diseño de políticas) Universidad de Costa Rica, Sistema de Estudios de Posgrado.

Lengyel, I., \& Molnar, E. (2015). The role of path dependence in the local economic transformation. J. Geogr. Inst. Cvijic., $65,373-390$.

MacKinnon, D., Cumbers, A., Pike, A., Birch, K., \& McMaster, R. (2009). Evolution in Economic Geography: Institutions, Political Economy, and Adaptation. Economic Geography, 85(2), 129-150.

Martin, E. (2004). Sustainable development, postmodern capitalism, and environmental policy and management in Costa Rica. Contemporary Justice Review, 7, 153-169.

Martin, R. (2008). Economic Geography: Critical Essays in Human Geography (Contemporary Foundations of Space and Place Series). Aldershot: Ashgate.

Martin, R., \& Sunley, P. (2006). Path dependence and regional economic evolution. European Science Foundation Exploratory Workshop on Evolutionary Economic Geography. Cambridge, U.K.: St Catherine's College - University of Cambridge.

Martin, R., \& Sunley, P. (2007). Complexity thinking and evolutionary economic geography. Journal of Economic Geography, 7, 573-601.

Martin, R., \& Sunley, P. (2010). The place of path dependence in an evolutionary perspective on the economic landscape. En R. Boschma, \& R. Martin, The Handbook of Evolutionary Economic Geography (págs. 62 - 92). Cheltenham, U.K.: Edward Elgar Publishing.

Merino, J. (2003). Cogeneración eléctrica favorece minorías. Revista de generación eléctrica (Federación costarricense para la conservación del ambiente).

Municipalidad de Pérez Zeledón. (2013). Acta No. 143-13. Obtenido de: http://feconcr.org/doc/represasur/ACTA-14313\%2829-01-2013\%29.pdf

Sanchez, A., Zúñiga, L., \& Cambronero, C. (2010). TLC y movimientos sociales: el movimiento contra el TLC en Costa Rica. Serie Sula Batsu, 1.

Smil, V. (2010). Energy transitions: histories, requirements, prospects. Westport, Ct.: Praeger Publishers.

Vargas, L. (2002). Competitiveness, innovation and democracy: Space for clean energy within electricity reforms. Heredia, Costa Rica: Editorial Fundación UNA.

Vargas, L. (2009). Producir más energía: cómo, cuándo y cuánto. Ambientales, 37, 3 - 22.

Wilde-Ramsing, J., \& Potter, B. (2008). Blazing the green path: Renewable energy and state-society relations in Costa Rica. The Journal of Energy and Development, 32, 68-90.

Witt, U. (2003). The Evolving Economy: Essays on the Evolutionary Approach to Economics. Cheltenham, U.K.: Edward Elgar Publishing. 